\title{
Visual search for a circular region perceived as a figure versus as a hole: Evidence of the importance of part structure
}

\author{
MARCO BERTAMINI and REBECCA LAWSON \\ University of Liverpool, Liverpool, England
}

\begin{abstract}
To study contour curvature polarity, we compared strictly convex regions (circular figures) with strictly concave regions (circular holes). We tested for an asymmetry between visual searches for concavities and those for convexities. We found that providing a preview of the background benefited search for concavities (holes) more than it did search for convexities (figures) and that for convex figures, nearer targets were responded to more quickly. Importantly, however, we failed to find any support for the hypothesis that concave targets are inherently more salient. We conclude that previous findings in the literature, which have been taken to indicate preferential processing of concavities, due to their increased salience, are more likely the result of an early computation of part structure based on concavities.
\end{abstract}

There is considerable interest in the study of contour curvature and contour curvature polarity. In this study, we were interested in what makes concavities special and whether they act as basic features in visual search. To compare strictly convex regions with strictly concave regions, we exploited the special properties of holes, as regions within a larger surface (Bertamini \& Croucher, 2003; Bertamini \& Mosca, 2004). To anticipate our conclusions, we found that concave contours are important for parsing complex shapes into parts but that they are not inherently more salient than convexities (Barenholtz, Cohen, Feldman, \& Singh, 2003; Humphreys \& Müller, 2000). This was the central research question of the study, but in addition, our experiments also support the principle of unidirectional border ownership and an advantage in searching for near targets, as opposed to far targets.

In the Gestalt tradition, convexity is discussed as a factor that contributes to figure-ground organization: Convex regions tend to be perceived as figures, and concave regions as background (Rubin, 1921; for empirical studies, see Kanizsa, 1979; Kanizsa \& Gerbino, 1976). The more recent literature has concentrated on convexity as a local property of a contour (Feldman \& Singh, 2005; Hoffman \& Richards, 1984; Koenderink, 1984). However, figure-ground organization is also critical in coding convexity and concavity. The process of segmenting an image implies a distinction between the inside and the outside of a region, which are seen as figure and background, respectively. On the basis of which side is perceived as the inside, the curvature along the contour has not only a

Address correspondence to M. Bertamini, Department of Psychology, University of Liverpool, Eleanor Rathbone Building, Bedford Street South, Liverpool L69 7ZA, England (e-mail: m.bertamini@liv.ac.uk). magnitude, but also a sign: positive for convex regions and negative for concave regions. This difference in the sign of the curvature is also referred to as contour curvature polarity (e.g., Fantoni, Bertamini, \& Gerbino, 2005).

One reason why contour curvature polarity is important is that, because of how objects self-occlude, contour polarity is informative about solid shape (surface curvature; Koenderink, 1984; Richards, Koenderink, \& Hoffman, 1985). Moreover, Hoffman and Richards (1984) have shown that when arbitrary solid shapes meet and interpenetrate, they form concave creases. For a smooth surface, the points along the crease are saddle points that can be seen in the image as concavities, and along a crease the curvature reaches a minimum. According to Hoffman and Richards (1984), visual shapes are composed of parts separated by minima of curvature. This part decomposition can contribute to how we recognize a complex shape on the basis of its structural description (Biederman, 1987; Marr \& Nishihara, 1978).

In the next two sections, we will review some empirical findings concerning differences between convexities and concavities. Both convexity and concavity advantages have been found, depending on the task.

\section{Evidence for a Processing Advantage for Concavities Relative to Convexities}

Hulleman, te Winkel, and Boselie (2000) and Humphreys and Müller (2000) tested visual search for simple, novel stimuli, examples of which are shown in Figure 1. Hulleman et al. found that search is more efficient when the target shape has a concavity than when it is strictly convex. ${ }^{1}$ Specifically, a visual search asymmetry (Treisman \& Souther, 1985) is present: Searching for a concavity among convex shapes is faster and is affected less by set size than is searching for a convex shape among shapes 
Hulleman et al. (2000)

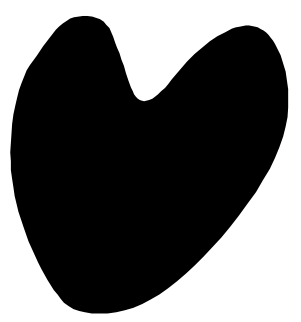

Concave target/distractor

Humphreys \& Müller (2000)

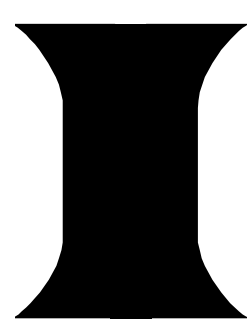

Concave target/distractor

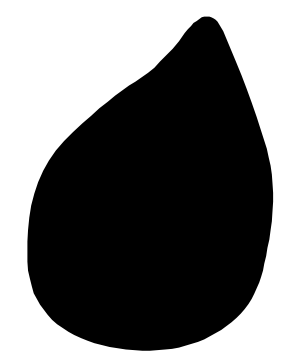

Convex target/distractor
Figure 1. Examples of stimuli similar to those used by Hulleman, te Winkel, and Boselie (2000) and Humphreys and Müller (2000). These stimuli have been redrawn for illustration. Area was matched for the concave and convex shapes. Curvature is matched at corresponding concave and convex locations. In both articles, the task was a visual search, and an asymmetry was found, with an advantage in searching for the concave target among convex distractors.

with concavities. In a series of experiments, they ruled out possible confounds and argued that concavities should be considered as basic features. Concavities may be more salient than convexities, due to their special status in defining the parts of an object (Hoffman \& Richards, 1984).

Humphreys and Müller (2000) performed similar experiments and confirmed the concavity advantage and the search asymmetry. Furthermore, they manipulated the figure-ground organization of the stimuli by closing the contours differently (see Figure 1). By changing figure-ground, it is possible to reverse contour polarity without changing the central pair of contours. The task remains unchanged, whereas the change in how contours are organized into figures and ground should be irrelevant if visual search is based only on edges. However, because a reversal of figure-ground implies a reversal of contour polarity, this might reverse the search asymmetry. This is what they found, supporting the idea that contour polarity is the critical factor. Humphreys and Müller's interpretation was similar to that of Hulleman et al. (2000). They argued that concavities can be selected quickly, perhaps via the activation of specialized detectors. We will refer to this idea that concavities are selected more quickly than convexities as the concavity hypothesis. Hulleman and Humphreys (2005) also reported that search performance is more efficient among figures than among holes. This finding is interesting but less relevant to the present set of experiments, because it does not compare convexity and concavity directly.

Barenholtz et al. (2003) reported results consistent with the concavity hypothesis and with the visual search data of Hulleman et al. (2000) and Humphreys and Müller (2000). They used a change detection task in which a polygon changed from one interval to the next. The change was equal in size; however, in one condition, it created an additional convexity, and in another condition, it created an additional concavity. Using a signal detection analysis and comparing $d^{\prime}$ values, Barenholtz et al. found that change detection was easier for the appearance of a concavity. The same was true when convexities and concavities were removed, instead of introduced. Barenholtz et al. suggested that concavities may be "inherently more salient" (p. 7).

There is, therefore, an advantage both for finding a concavity in a visual search task and for detecting the appearance or disappearance of a concavity in a change detection task. In theory, preattentive and attentive processes might differ. It is, therefore, interesting that evidence has been interpreted as indicating a privileged detection of concavities for both preattentive (visual search) and attentive (change detection) tasks. However, Bertamini and Farrant (2005) replicated Barenholtz et al.'s (2003) results but found that it was the change of sign that was easy to detect, rather than the introduction of a concavity per se. That is, with the change detection paradigm, a new convexity in the context of a concave contour is just as salient as a new concavity in the context of a convex contour. ${ }^{2}$

We believe that the concavity hypothesis is not the only interpretation of these results. Hulleman et al. (2000), Humphreys and Müller (2000), and Barenholtz et al. (2003) all mentioned an alternative account of their findings: Concavities may be selected more quickly or be more salient because of their influence on perceived part structure. Looking at Figure 1, the concavities, unlike the convexities, appear to split the shapes into two subparts, making it possible for observers to search for the more complex (multipart) target (see Rosenholtz, 2001). A clear parallel exists with the classic example of search asymmetry in which a $\mathrm{Q}$ is found among Os more easily than vice versa (Treisman \& Souther, 1985). We call this alternative account the part structure hypothesis. We will turn now to evidence consistent with the part structure hypothesis.

\section{Effects of Contour Polarity Mediated by Perceived Part Structure}

Several studies have shown a convexity advantage in the time taken to judge the position of vertices perceived as either convex or concave (Bertamini, 2001; Bertamini \& Croucher, 2003; Bertamini, Friedenberg, \& Argyle, 2002; Bertamini \& Mosca, 2004; Gibson, 1994). Bertamini suggested that positional information is more readily available for vertices perceived as convex because parts have 
a position in a way that boundaries between parts (i.e., concavities) do not.

Perhaps the most convincing example of this convexity advantage has come from studies in which the same region has been presented as a figure in some trials and as a hole in other trials (Bertamini \& Croucher, 2003; Bertamini \& Mosca, 2004). When observers judge the relative position of the two outside vertices of an irregular hexagon, they are faster when the vertices are convex (pointing outward) than when they are concave (pointing inward, creating an hourglass shape). However, when the same irregular hexagons are perceived as holes, the relative performance is reversed, with faster judgments to convex (now inwardpointing) vertices. This is important because the information in terms of contours per se is identical in both instances and is sufficient to select the correct response. ${ }^{3}$

\section{Comparing Search for Convexities and \\ Concavities}

We aim to resolve the differences between these two sets of empirical results, the first of which shows a concavity advantage (supported by a reversal of this advantage with figure-ground reversal; see, e.g., Humphreys \& Müller, 2000) and the second of which shows a convexity advantage (again supported by a reversal of the effect with figure-ground reversal; see, e.g., Bertamini \& Croucher, 2003). If concavities are inherently more salient than convexities or can be classed as basic features, one would expect better performance with concavities than with convexities, whatever the task. Alternatively, the findings could be due to changes in part structure, rather than to concavities per se. Concavities are used by the visual system to parse a shape into subparts, and this process may be both fast and obligatory (Singh \& Hoffman, 2001). To differentiate between these two accounts, stimuli that consist solely of either convexities or concavities are needed. The concave stimuli presented in earlier studies always also included convexities (see Figure 1). In contrast, if a contour is strictly concave, the process of contour analysis will not find parts punctuated by the change in the sign of the curvature. The part structure hypothesis claims that it was the change from positive to negative contour polarity (i.e., from convexity to concavity), signaling a boundary between parts, rather than the presence of negative curvature per se, that produced the search asymmetries (an advantage for detecting concave targets) discussed above (see also Bertamini \& Farrant, 2005).

One potential source of confusion in the interpretation of the findings about contour polarity is the meaning of the words convex and concave when applied to contours and when applied to objects or closed shapes. It is possible to have local convexity and local concavity, but with respect to an object, curvature can be strictly positive but cannot be strictly negative. A strictly negative contour defines a hole, not an object (for the study of the perception of holes, see Bertamini \& Croucher, 2003; Nelson \& Palmer, 2001; Palmer, 1999, pp. 285-287). In the visual search experiments discussed above (Hulleman et al., 2000; Humphreys \& Müller, 2000), the concave shapes included convexi- ties, whereas the convex shapes had no concavities (see Figure 1). If concavities create parts by specifying part boundaries, the concave shape had multiple parts, whereas the convex shape had only one part. Even with the figureground reversal introduced by Humphreys and Müller, the concave stimuli had more parts (four) than did the corresponding convex stimuli (two). It may be that concavities are responsible for the visual search asymmetry (Hulleman et al., 2000; Humphreys \& Müller, 2000), but this effect is mediated by the role that they have in part decomposition when they alternate with convexities. ${ }^{4}$

As soon as a new concavity is introduced into an object, the resulting object may be perceived as having a more complex part structure. To avoid this problem, we compared circular figures (convex curvature) and circular holes (concave curvature) to test the predictions of the concavity hypothesis and the part structure hypothesis. Circular figures and holes also have the advantage of having no curvature extrema. For the purposes of this study, it was important that the contour of a hole should belong to the surrounding object. We make no specific claims about the more general issue of how the shape of a hole is perceived (Palmer, 1999). It is worth noting that other hypotheses could be formulated. For instance, a surface with a hole and a surface without a hole could be described as having different part structures. This is not our chosen meaning of part structure, because we have restricted it to the case of a parsing along contours triggered by the change from positive to negative curvature. We treat the difference in shape between figures and figures with holes as a topological difference that might, in itself, affect visual search, but our prediction is that it will not do so.

We report a series of seven experiments in which we compared strictly convex items (circular figures) and strictly concave items (circular holes), using the visual search paradigm. When our experiments were already underway, we discovered that similar experiments had already been published, in an attempt to answer a different question. O'Toole and Walker (1997) examined whether a search asymmetry existed for items defined by crossed or uncrossed disparity. This is similar to our Experiments 1 and 2 , in that when regions of random-dot stereograms are assigned crossed and uncrossed disparity, they are inevitably perceived as figures (in front of the background at fixation) and holes (behind the background at fixation), respectively. O'Toole and Walker's conclusions were mixed, but there was a trend for a search asymmetry in favor of figure targets and in favor of targets in front, relative to targets behind (relative to distractors). One of the complications was the presence of nonlinear trends in the search slopes. We will return to consider the relationship of our findings with those obtained by O'Toole and Walker.

\section{GENERAL METHOD}

\section{Participants}

Observers were recruited from the staff and students at the University of Liverpool. They were naive with respect to the hypotheses until after the data had been collected. 


\section{Stimuli}

The stimuli were generated on a Macintosh G4 computer and presented on a Sony F500T9 monitor with a resolution of $1,280 \times$ 1,024 pixels at $120 \mathrm{~Hz}$. Two stereo images were presented with the use of a NuVision infrared emitter and stereoscopic glasses. The effect of interleaving left and right images was that effective vertical resolution and refresh rate were halved $(512$ pixels at $60 \mathrm{~Hz})$. The computer recorded whether each response was correct and the reaction time (RT) in milliseconds, using Video Toolbox functions (Pelli, 1997).

There was an $18 \times 18 \mathrm{~cm}$ rectangular background of random dots on which up to nine circular regions $(1.63-\mathrm{cm}$ diameter) were placed (see Figure 2). For Experiments 1, 2, 3, and 5, the location of each of these circular target and distractor regions was chosen randomly within a $3 \times 3$ matrix $(8.4 \times 8.4 \mathrm{~cm})$, with each location having a random shift in both $x$ and $y$ of up to $0.3 \mathrm{~cm}$. The observers were approximately $57 \mathrm{~cm}$ from the screen, so $1 \mathrm{~cm}$ on the screen equaled $1^{\circ}$ of visual angle. For Experiments 4 and 6, the items were slightly smaller (diameter, $1.46 \mathrm{~cm}$ ). They were placed within a $4 \times 4$ matrix
$(9.0 \times 9.0 \mathrm{~cm})$, and each location had a random shift in both $x$ and $y$ of up to $0.3 \mathrm{~cm}$. On each trial in every experiment, new random-dot patterns were generated for both the background and the items, and new random locations of the items were used.

\section{Procedure}

We administered the TNO stereotest to all the participants as a screening tool and recorded their thresholds. These values ranged between 15 and 120 arcsec. The observers sat in a dimly illuminated room. They held a game pad and were instructed to press one button if the target was present and a different button if the target was absent. Once the session started, 24 trials formed a practice phase. Half of the participants in each experiment searched for convex targets (i.e., figures) in the first block and then searched for concave targets (i.e., holes) in the second block. For the other half of the participants, the order of the blocks was reversed.

Each participant responded to a total of 720 experimental trials The trials were presented in rapid succession, but after every 90 trials, the observer was allowed a self-timed rest.

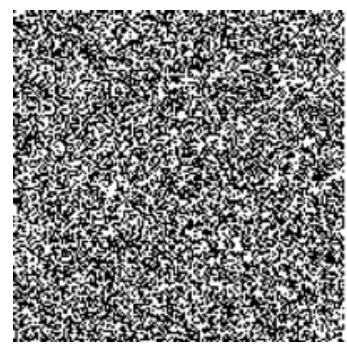

Left eye

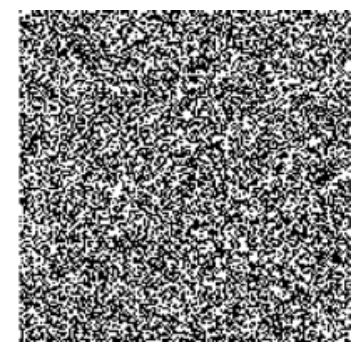

Right eye
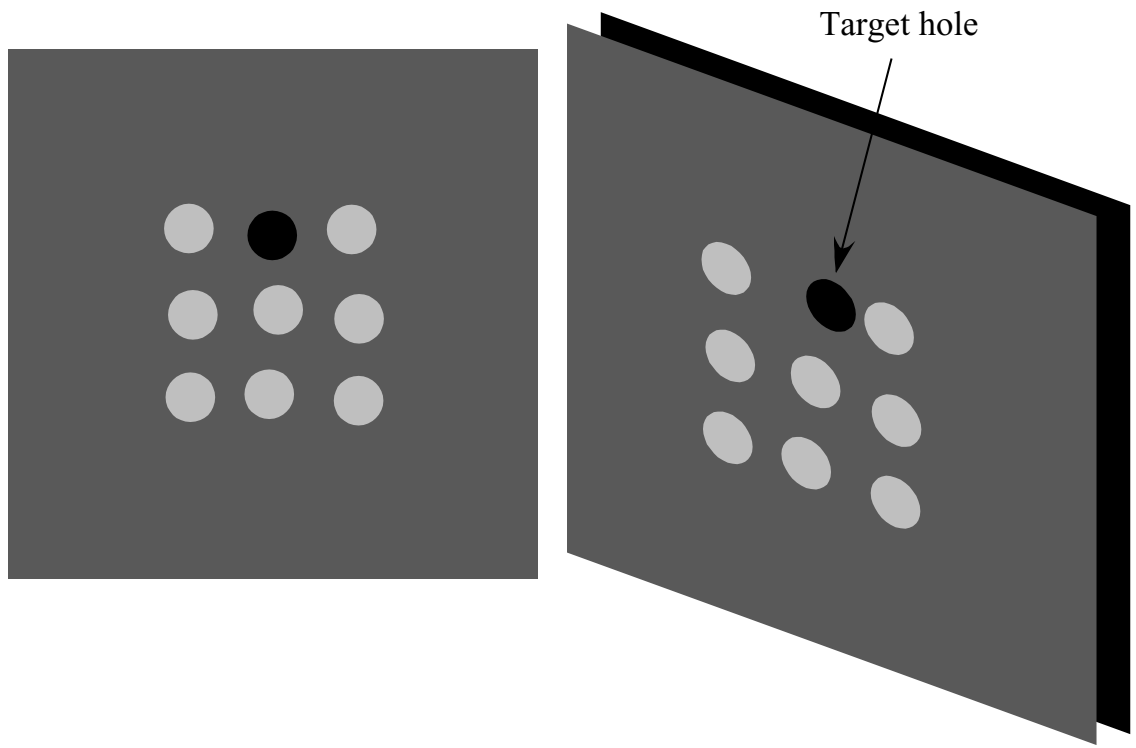

Figure 2. The stimuli used in Experiments 1 and 2. (Top) A pair of random-dot stereograms with eight figure distractors and a target hole. In this example, some of the background has been cropped, and the disparity increased to allow the reader to cross-fuse the two images. (Bottom) Diagram illustrating the stimuli, with the size of the background, the items, and the random $x$ and $y$ offset to scale. The nine items set size condition is used as an example, with stimuli at all available locations in an invisible $3 \times 3$ matrix. In this and in the following figures, middle gray represents the zero-disparity plane, darker regions have uncrossed disparity, and lighter regions have crossed disparity. The diagram on the left illustrates the fact that item contours are identical if one does not take distance in depth into account. The diagram on the right is an attempt to convey the depth dimension. 


\section{EXPERIMENT 1 Visual Search for a Figure or for a Hole}

In this experiment, the task was a visual search for either a target figure among distractor holes or a target hole among distractor figures. The stimuli used by Hulleman et al. (2000) and by Humphreys and Müller (2000) had concavities present within complex shapes with alternating convex and concave regions (see Figure 1). In the present experiment, we compared a strictly convex target and a strictly concave target: A circular figure has a contour that is completely convex, and a circular hole has a contour that is completely concave. According to the concavity hypothesis, concave contours are inherently more salient. Therefore, first, faster responses would be predicted for hole targets than for figure targets (i.e., a main effect of type). Second, a search asymmetry was predicted in favor of finding the hole target (an interaction between set size and type, with the advantage for hole over figure targets increasing at larger set sizes). In contrast, according to the part structure hypothesis, since both the figure and the hole stimuli were simple circles, in neither case was there a change from positive to negative curvature along the contour. Therefore, no main effect of type would be predicted, and no search asymmetry was expected.

\section{Method}

Participants. Twenty students participated. For 10 participants, the disparity was set at 9.99 arcmin. For the other 10, the disparity was set at 3.33 arcmin.

Design. The factors were target type (figure vs. hole), target presence (present vs. absent), and set size (3, 6, or 9 items), which were factorially combined in a within-subjects design.

\section{Results and Discussion}

Mean RTs and slopes are shown in Figure 3. Overall error rate was $3.1 \%$, so the statistical analyses focused on RT. The analysis was performed on correct trials with RTs below 4,000 msec; longer RTs constituted less than $1 \%$ of the data. There were three within-subjects factors: target type (hole or figure), target presence (present or absent), and set size (3, 6 , or 9 items). Here and in all the subsequent analyses, we entered set size as a linear contrast, so as to treat the effect as a linear slope. As can be seen in Figure 3, the assumption of linearity is reasonable. There were also two betweensubjects factors: disparity (3.33 or 9.99 arcmin) and order (search for a figure first or search for a hole first).

A mixed repeated measures ANOVA (type $\times$ presence $\times$ set size $\times$ disparity $\times$ order) on RT yielded significant main effects of type $[F(1,16)=6.90, p=.018]$, presence $[F(1,16)=20.38, p=.001]$, and the linear contrast of set size $[F(1,16)=354.93, p=.001]$. Responses were faster when the target was a hole and when the target was present, and RT increased linearly with increases in set size. There were also significant interactions between target type and target presence $[F(2,16)=8.07, p=.012]$, with faster responses to target present for both figures and holes, but with a larger difference for figure targets, and between set size and target presence $[F(1,16)=5.36, p=$ $.034]$, but not between type and set size $[F(1,16)=3.41$, n.s.]. There were no significant effects of either disparity or order, and these between-subjects variables did not interact with any of the within-subjects variables.

If we take a significant difference in slopes as the necessary criterion for a search asymmetry (i.e., a type $\times$ set size interaction), there was no search asymmetry in this experiment, consistent with the predictions of the part structure hypothesis, and contrary to the predictions of the concavity hypothesis. However, there was a main effect of type, supporting the prediction of the concavity hypothesis that it would be easier to find a concave target: On average, the hole target advantage was $33 \mathrm{msec}$. However, this concavity advantage is the opposite of that found by O'Toole and Walker (1997). After careful consideration of the differences between our parameters and those used by O'Toole and Walker, we formulated a hypothesis to account for this difference between the two studies, which was tested in Experiment 2.

\section{EXPERIMENT 2 Effect of Removing the Background Preview}

Experiment 1 did not show a search asymmetry: There was no interaction between type and set size, supporting the part structure hypothesis. However, the main effect of type could be taken as supporting the concavity hypothesis that concavities are more salient. Experiment 1 did not, therefore, fully support either of the two alternative hypotheses outlined in the introduction. In addition, Experiment 1 failed to replicate O'Toole and Walker's (1997) results.

Understanding the reason for this discrepancy could clarify the interpretation of the results of Experiment 1. Several minor aspects of the stimuli and the procedure differed between the two studies. For instance, we used circular stimuli, whereas they used squares. We hypothesized that the most important difference may have been that the stimulus background was presented to our observers for $666 \mathrm{msec}$ before the stimuli appeared in each trial, whereas the background and the stimuli were presented simultaneously in O'Toole and Walker's (1997) study. Preview of the background in Experiment 1 may have meant that the detection of a hole required only the detection of a change in the shape of an object (the background) that the observer was already monitoring. However, detection of a figure required the detection of a new surface unrelated to the background. This difference may have caused the faster responses to hole targets in Experiment 1. To test this proposal, Experiment 2 replicated Experiment 1, but with no preview of the background.

\section{Method}

The method and procedure were identical to those in Experiment 1, except for two changes. First, both background and the items appeared simultaneously in Experiment 2. Second, since there was no effect of disparity in Experiment 1, we used only the value of 3.33 arcmin in Experiment 2. Ten observers participated.

\section{Results and Discussion}

Mean RTs and slopes are shown in Figure 3. We performed an analysis similar to that in Experiment 1. Over- 


\section{Experiment 1}

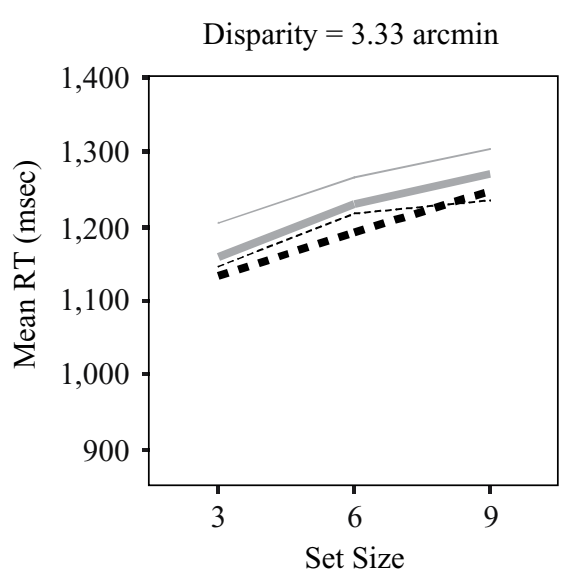

Figure slope: $18.07 \mathrm{msec} /$ item Hole slope: $17.09 \mathrm{msec} /$ item

Experiment 2

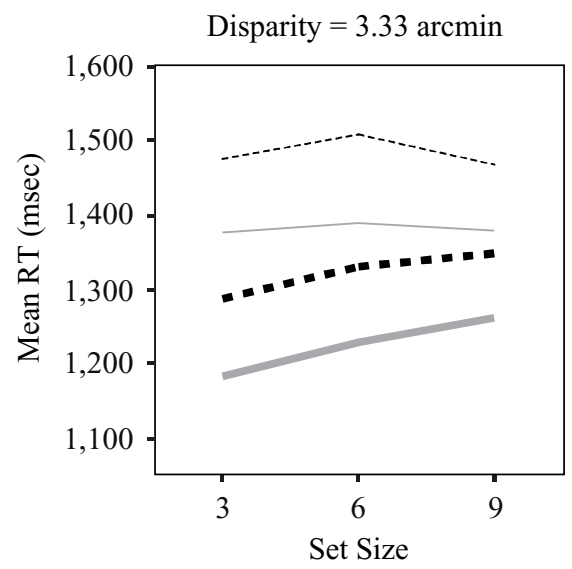

Figure slope: $13.13 \mathrm{msec} /$ item Hole slope: $10.37 \mathrm{msec} /$ item

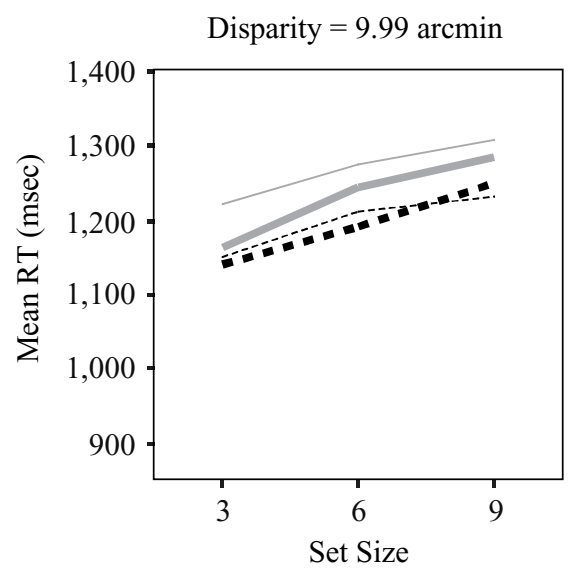

Figure slope: $22.23 \mathrm{msec} /$ item Hole slope: $17.77 \mathrm{msec} /$ item

$$
\begin{aligned}
& \text { Figure present } \\
& \text { - - Hole present } \\
& \text { - Figure absent } \\
& \ldots \text { - Hole absent }
\end{aligned}
$$

Figure 3. Results from Experiment 1 (top) and Experiment 2 (bottom). Mean reaction time (RT) is plotted against set size for the two types of targets (figure and hole, coded as solid and dashed lines, respectively) and for target-present and target-absent trials (thinner lines indicate target absent). The slopes for target-present trials, in milliseconds/item, are given below each graph for the searches for figures and for holes. For Experiment 1, the left and right graphs plot data for the two versions of the experiment with different values of binocular disparity (3.33 and 9.99 arcmin). Omnibus within-subjects standard errors of the means were computed under the assumption of sphericity; they were 18.68 and $18.80 \mathrm{msec}$, respectively, for the two versions of the experiment. For Experiment 2, only the smaller, 3.33arcmin disparity was used, and the omnibus within-subjects standard error of the mean was 52.29 msec.

all error rate was $2.9 \%$. There were three within-subjects factors: target type (hole or figure), target presence (present or absent), and the linear contrast of set size $(3,6$, or 9 items). There was one between-subjects factor: order (search for a figure first or search for a hole first).

A mixed repeated measures ANOVA (type $\times$ presence $\times$ set size $\times$ order) on RT yielded significant main effects of type $[F(1,8)=6.10, p=.039]$ and presence
$[F(1,8)=10.68, p=.011]$ and a marginal effect of set size $[F(1,8)=5.16, p=.053]$. Responses were faster when the target was a figure and when the target was present, and RT increased linearly with increases in set size. There was also an interaction between set size and target presence $[F(1,8)=6.69, p=.032]$, but importantly, there was no interaction between type and set size $[F(1,8)=$ 1.05, n.s.]. 
In Experiment 2, the effect of type changed from an advantage for holes to an advantage for figures (see Figure 3). We believe that the reason for this is that in Experiment 1, the onset of a hole target changed the shape of the previewed background surface (from a background to a background with a hole), whereas the onset of the figure target did not. In contrast, in Experiment 2, the background surface was presented at the same time as the targets and distractors, so the background did not change shape during the trial and, hence, there was no advantage for the detection of hole targets versus figure targets. This argument does not explain the figure advantage in Experiment 2; this may have been due to their relative distance in depth, relative to the distractors. We tested this idea in Experiment 3.

It is important to note that this interpretation of the difference between Experiments 1 and 2 is true only if one accepts the principle of unidirectional border ownership. In terms of local change, the new patch of random dots that appeared was the same for the figures and the holes and was not simply a displacement of dots that were already part of the background. We did this because we wanted to make sure that what was perceived was a series of independent surfaces at different distances in depth from the observer. Locally, the only difference between the figures and the holes was the direction of the disparity in both Experiments 1 and 2. To understand the difference in outcome between the two experiments, one must think of the holes as surrounded by contours belonging to the background surface, whereas the figures own their own contours. Only when the contours around the hole belong to the background is it correct to claim that holes have concave curvature. In other words, the border is not owned by the holes per se.

\section{EXPERIMENT 3 Search for Targets in Front or Behind (Relative to the Distractors)}

In Experiments 1 and 2, figures and holes were created by assigning crossed and uncrossed disparity, respectively, to small regions of random dots. This meant that contour curvature polarity was confounded with type of disparity (crossed and uncrossed) and also with perceived egocentric distance, because the figures were nearer than the background, whereas a surface farther than the background was seen through the holes. In Experiment 3, we examined how important these factors were by replicating Experiment 2 but removing the background altogether. In contrast to the random-dot stereograms presented in Experiments 1 and 2 , the stimuli were random dot circular patches on a uniform gray background, so that all the stimuli appeared to be figures (none would be perceived as holes) and only disparity separated the targets from the distractors. In one version of the experiment, we compared search for a target with crossed disparity among distractors with uncrossed disparity with the reverse situation. In another version of the experiment, we compared search for a target that had a larger crossed disparity among distractors that had a smaller crossed disparity with the reverse situation. These two versions of the experiment tested how important it is to straddle the zero disparity plane. If the results from the two versions are similar, we can conclude that the distance factor (nearer vs. farther) is more important than the type of disparity (crossed vs. uncrossed). If in both experiments there is an advantage for responding to targets in front of distractors, this could explain the main effect of type (faster responses to the nearer figure targets than to the more distant hole targets) found in Experiment 2.

\section{Method}

The method and procedure were identical to those in Experiment 2, except that a uniform gray region with a 1-pixel black outline replaced the random-dot background (see Figure 4). We included the outline in order to make the task easier by providing some information about the location of the zero-disparity plane, but we do not believe that this was a critical aspect of the display. Twenty observers participated. Ten were assigned to the crossed-disparity-only version, and 10 to the crossed-versus-uncrossed-disparity version.

\section{Results and Discussion}

Mean RTs and slopes are shown in Figure 4. We performed an analysis similar to that in Experiment 1. Overall error rate was $6.7 \%$. There were three within-subjects factors: target distance (in front or behind), target presence (present or absent), and the linear contrast of set size (3, 6, or 9 items). There were two between-subjects factors: order (search for a target in front first or search for a target behind first) and version (both crossed and uncrossed disparities presented or only crossed disparities presented).

A mixed repeated measures ANOVA (distance $\times$ presence $\times$ set size $\times$ order $\times$ version) on RT yielded significant main effects of presence $[F(1,16)=24.24, p=.001]$ and of set size $[F(1,16)=32.35, p=.001]$. Responses were faster when the target was present, and RT increased linearly with increases in set size. Although distance in depth was not significant, there was a significant interaction between distance and set size $[F(1,16)=5.59, p=$ $.031]$. As can be seen in Figure 4, slopes were steeper when the targets were behind, rather than in front. There was also a significant interaction between set size and target presence $[F(1,16)=9.29, p=.008]$. Version was not significant and did not interact with any other factor.

Experiment 3 provided evidence for a search asymmetry, with faster search for targets in front, rather than behind. This factor may, therefore, have produced the convexity (figure target) advantage that we found in Experiment 2. These results further suggest that relative distance in depth (in front vs. behind) is more important than whether binocular disparity is crossed or uncrossed, since no difference was found between the two versions of the experiment (but note that the former was a within-subjects factor and the latter a between-subjects comparison). The in-front advantage is consistent with the finding that when attention is allocated in 3-D, its spread in depth is not equal (Andersen, 1990) and that reallocation of attention is faster when observers switch attention from a far to a near location than when they switch attention from a near to a far location (Arnott \& Shedden, 2000; Downing \& Pinker, 1985). A recent article in the context of shape from shading by Kawabe and Miura (2004) has also shown that search is easier 


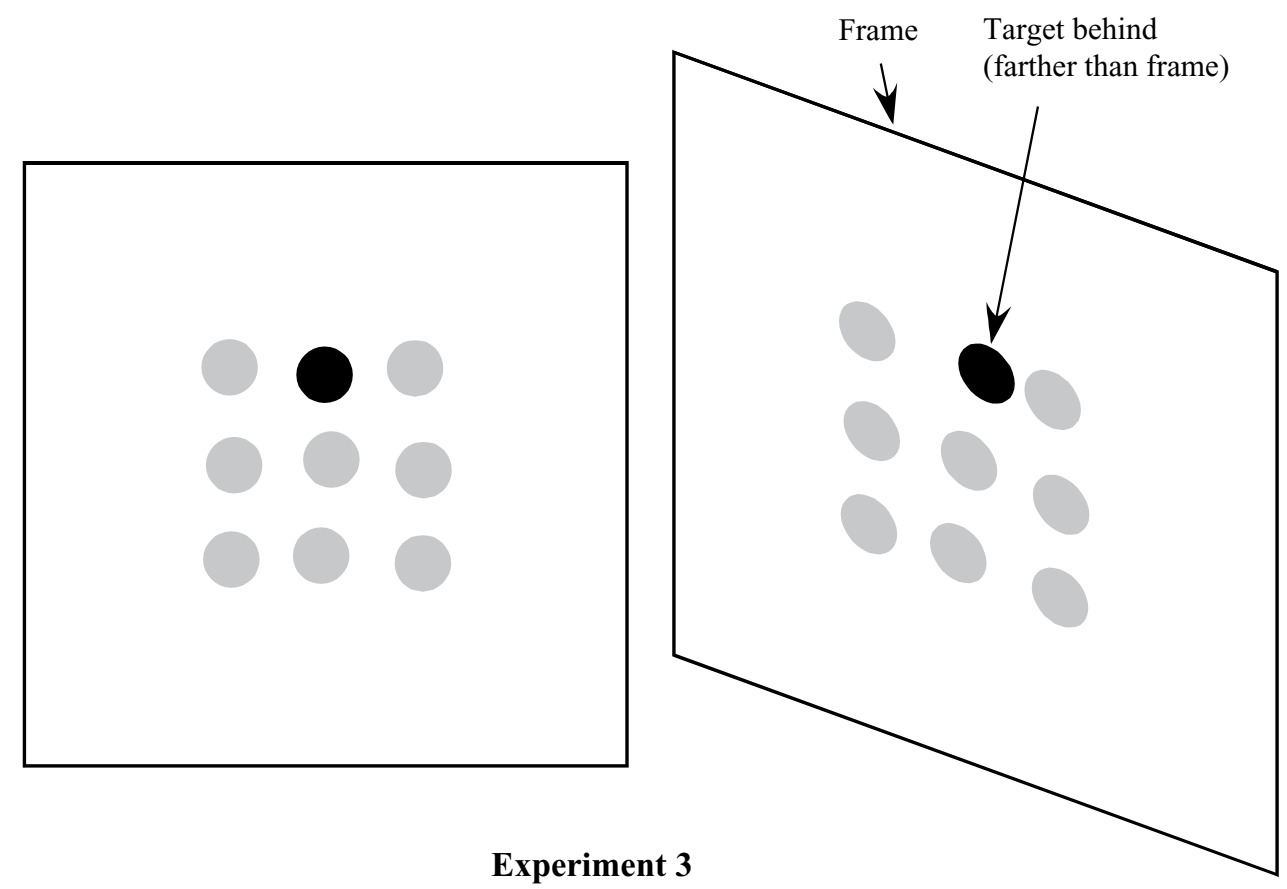

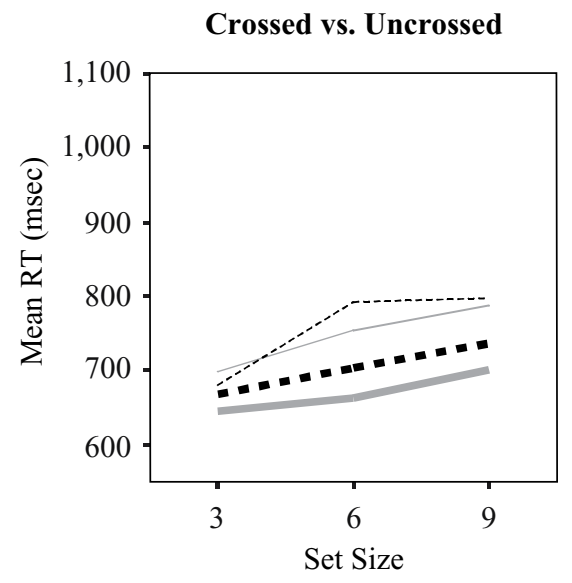

In front slope: $9.28 \mathrm{msec} /$ item Behind slope: $11.46 \mathrm{msec} /$ item

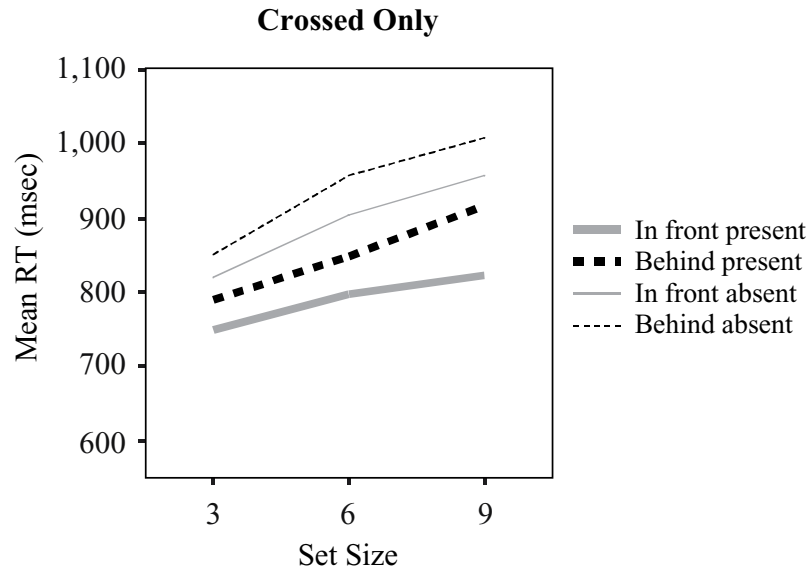

In front slope: $12.57 \mathrm{msec} /$ item Behind slope: $21.16 \mathrm{msec} /$ item

Figure 4. The stimuli (with target behind) and results from Experiment 3. Mean reaction time (RT) is plotted against set size for the two types of targets (targets in front and targets behind, relative to the distractors) and for target-present and target-absent trials. Slopes for target-present trials, in milliseconds/item, are given below each graph for searches for targets in front and targets behind. The left and right graphs plot data for crossed versus uncrossed disparity and for crossed disparity only (near vs. far), respectively. The omnibus within-subjects standard errors of the means were 26.93 and $16.91 \mathrm{msec}$, respectively.

for a shape formed by convex mounds, rather than by concave dimples. Interestingly, the convex mounds were also judged nearer than the concave dimples.

\section{EXPERIMENT 4 Sparse Display}

The concavity hypothesis predicted faster search for holes (concave targets) than for figures, particularly at larger set sizes. In neither Experiment 1 nor 2 was there a significant interaction between type and set size of that nature. We are, therefore, inclined to conclude that concavities as such do not behave as basic features that are so salient that they can be detected preattentively. This is consistent with our preferred interpretation of the literature and with the part structure hypothesis.

On the other hand, we are basing our conclusion on null findings, and it is, therefore, important to replicate and extend our results. In O'Toole and Walker (1997), the authors found that the type of display and the number 
of items used affected the outcome. We agree with their speculation that item configurations may appear regular with large set sizes and, hence, these conditions may not be as difficult as expected, because the items form a close, homogeneous structure and, perhaps, even a new surface. In O'Toole and Walker, this factor led to some nonlinearity in the search slopes. We saw no evidence of nonlinearity in the present studies. Nevertheless, if slopes did flatten at larger set sizes, it would be harder to find significant slope differences. Because of these concerns, in Experiment 4 we replicated Experiments 1 and 2, with greater variability in the location of the items. This was achieved by placing items within a larger, invisible $4 \times 4$ matrix (instead of a $3 \times 3$ matrix) and making the items slightly smaller (see Figure 5). We replicated Experiments 1 and 2 because of the unexpected but important differences in their results.

\section{Method}

The method and procedure were identical to those in Experiments 1 and 2. One version of Experiment 4 presented a preview of the background, and another version had no preview, as in Experiments 1 and 2, respectively. The stimuli were different because the 3,6 , or 9 items were randomly placed within an invisible $4 \times 4$ (rather than a $3 \times 3$ ) matrix (see Figure 5). Twenty-four observers participated. Twelve were assigned to the preview version, and 12 to the no-preview condition.

\section{Results and Discussion}

Mean RTs and slopes are shown in Figure 5. We performed an analysis similar to that in Experiment 1. Overall error rate was $2.7 \%$. There were three within-subjects factors: target type (hole or figure), target presence (present or absent), and the linear contrast of set size (3,6, or 9 items). There were two between-subjects factors: order (search for a figure first or search for a hole first) and background preview (present or absent).

A mixed repeated measures ANOVA (type $\times$ presence $\times$ set size $\times$ order $\times$ preview) on RT yielded significant main effects of type $[F(1,20)=24.48, p=$ $.001]$, presence $[F(1,20)=55.16, p=.019]$, and set size $[F(1,20)=137.23, p=.001]$. Responses were faster when the target was a figure and when the target was present, and RT increased linearly with increases in set size. Importantly, there was no interaction between type and set size $[F(1,20)=3.33$, n.s.]. Although background preview was not significant, there was a significant interaction between preview and type $[F(1,20)=13.32, p=$ $.002]$. As can be seen from Figure 5, the figural advantage was larger when there was no background preview. This effect was in the same direction as that observed in Experiment 1, in comparison with Experiment 2, but was not identical, since the preview did not lead to faster responses to the holes as targets. This is not too surprising, if the explanation for a hole advantage in Experiment 1 was the fact that the observers responded to a change in the shape of the background. In Experiment 4, the change was smaller (because all the items were smaller), and its location was less predictable (because of the sparseness of the display).
Experiment 4 therefore supports our interpretation of the results of Experiments 1 and 2: There was no concavity advantage when all the items and the background appeared simultaneously and no search asymmetry, since there was no interaction between type and set size. Observers can monitor for a change in the shape of the background when there is a background preview, making search for concave targets (holes) somewhat easier. This strategy may be adopted by some observers and not by others. Some evidence for this possibility was that individual variability was higher when the background preview was present.

\section{EXPERIMENT 5 Manipulation of Target Distance}

Experiments 1 and 2 compared figures and holes relative to a large background located at the zero-disparity plane. Inevitably, this meant that the figures were in front of the background and the region seen through the hole was farther away than the background. On the basis of the results of Experiment 3, it is possible that the advantage of figure targets, relative to hole targets, in Experiment 2 was at least partly an artifact of faster responses to targets in front (figures) than to targets behind (holes).

In Experiment 5, the stereoscopic distance of the target from the observer was manipulated, so that the target, whether a figure or a hole, could appear either in front or behind the other items (distractors; see Figure 6). To arrive at a situation in which both the figures and the holes could be placed at arbitrary distances in depth from the observer and with the same local disparity between the local background and the figure or hole, we used local reference backgrounds (for both the figures and the holes) and, for the holes only, local backgrounds, which were visible through the hole. What this meant was that for both figures and holes, a new surface was present locally, either on top of the local background or just behind it. ${ }^{5}$ There were no changes to the shape of the main background (cf. Experiment 1), because the local background appeared simultaneously with the items (figures and holes).

There are three possible scenarios. (1) If the in-front advantage had overshadowed a small concavity (hole) advantage in Experiments 2 and 4, Experiment 5 could, for the first time, confirm faster responses for concavities, as predicted by the concavity hypothesis. (2) If the convexity (figure) advantage found in Experiments 2 and 4 was due to an advantage for items in front, distance should affect performance in Experiment 5, but any difference between figures and holes should disappear, as predicted by the part structure hypothesis. (3) Conversely, if there is a convexity (figure) advantage unrelated to the advantage for targets in front, faster responses for figures should be found in Experiment 5, and distance should not affect performance. This convexity advantage would be the exact opposite of that predicted by the concavity hypothesis.

\section{Method}

The method and procedure have been described in the General Method section. Each item was presented together with a square 

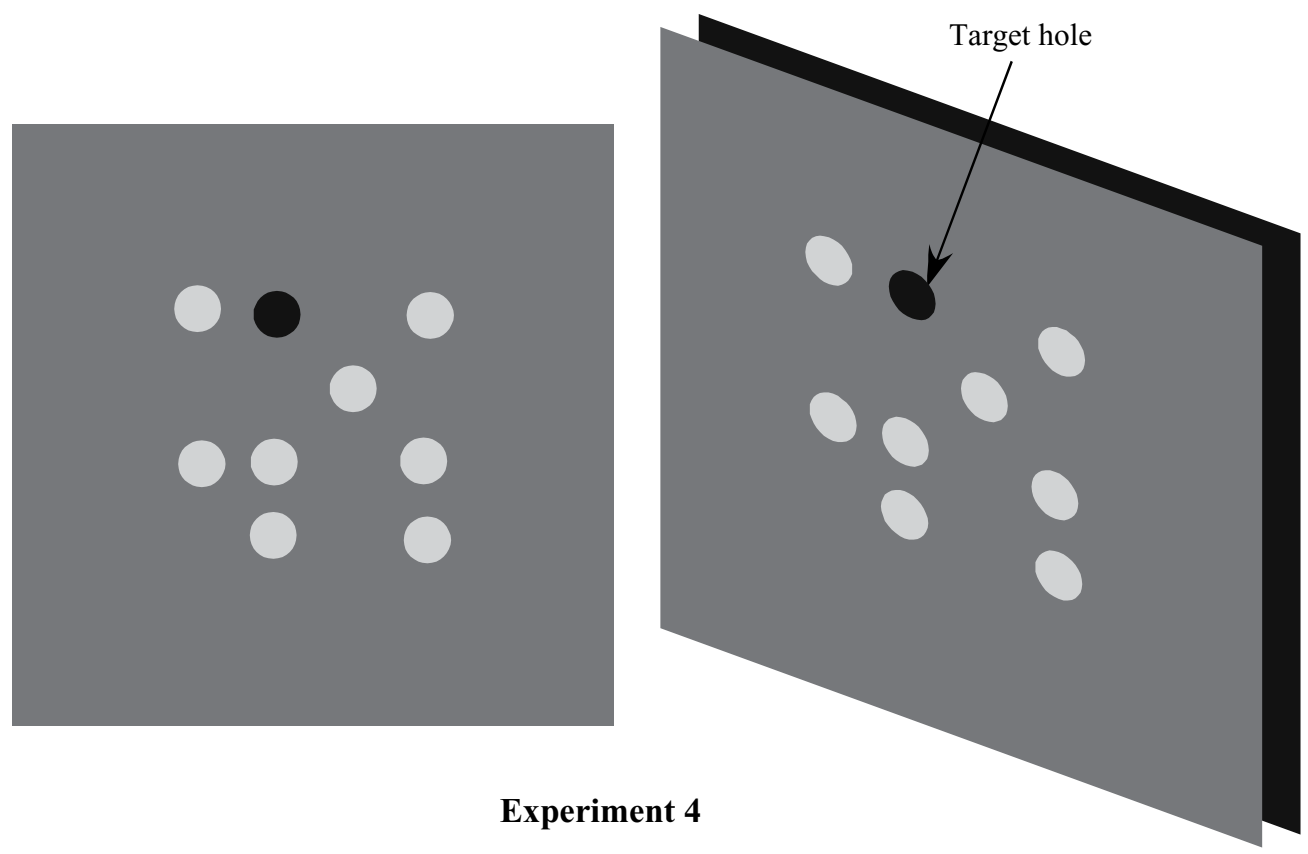

Experiment 4

With Background Preview

Without Preview

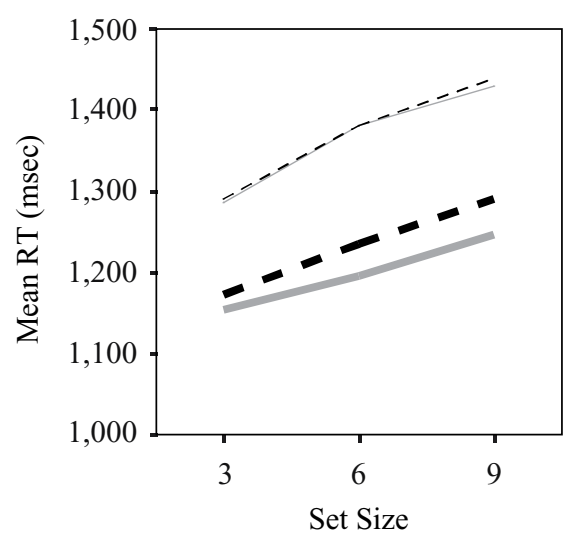

Figure slope: $15.64 \mathrm{msec} /$ item Hole slope: $19.81 \mathrm{msec} /$ item

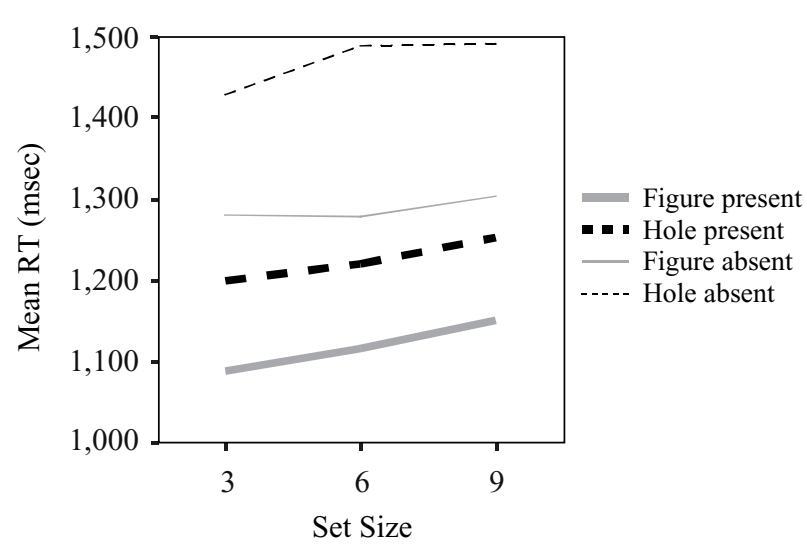

Figure slope: $10.15 \mathrm{msec} /$ item Hole slope: $9.02 \mathrm{msec} /$ item

Figure 5. The stimuli (for a hole target) and results from Experiment 4. Mean reaction time (RT) is plotted against set size for two types of targets (figure and hole) and for target-present and target-absent trials. The left and right graphs show the results when a preview of the background was either present or absent (omnibus within-subjects standard errors of the means were 28.89 and $36.30 \mathrm{msec}$, respectively). Slopes, in milliseconds/ item, are given below each graph for searches for figure targets and for hole targets.

region around it that we call the local background. The figures were seen in front of a local background. The holes were holes in a local background, through which another local surface was visible (see Figure 6).

The disparity of the surface with a circular contour (surface-withhole or circular figure) could be located on one of three different planes $(3.33,6.66$, or 9.99 arcmin). The disparity between the surface with a circular contour and the local background was always 3.33 arcmin. Ten observers participated.

\section{Results and Discussion}

Mean RTs and slopes are shown in Figure 6. We performed an analysis similar to that in Experiment 1. Over- all error rate was $4.5 \%$. There were four within-subjects factors: target type (hole or figure), target presence (present or absent), the linear contrast of set size (3, 6, or 9 items), and target distance (near, intermediate, or far). There was one between-subjects factor: order (search for a figure first or search for a hole first).

A mixed repeated measures ANOVA (type $\times$ presence $\times$ set size $\times$ distance $\times$ order) on RT yielded significant main effects of presence $[F(1,8)=8.64, p=.019]$ and of set size $[F(1,8)=36.90, p=.001]$. Responses were faster on target present trials, and RT increased linearly with increases in set size. The interaction between 

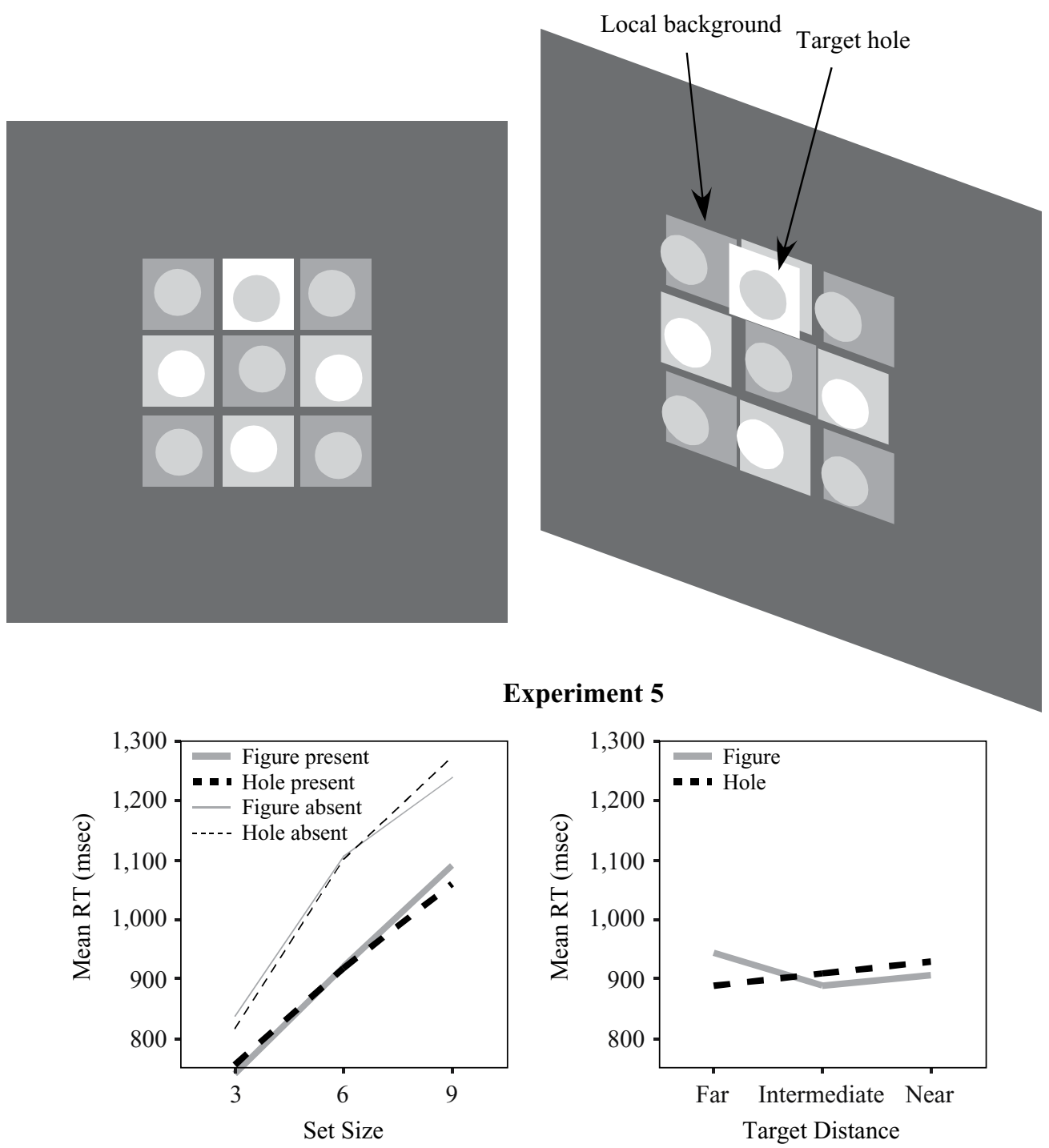

Figure slope: $58.46 \mathrm{msec} /$ item

Hole slope: $51.04 \mathrm{msec} /$ item

Figure 6. The stimuli (for a hole target) and results from Experiment 5. On the left, mean reaction time (RT) is plotted against set size for the two types of targets (figure and hole) and for target-present and target-absent trials. The omnibus within-subjects standard error of the mean was $18.68 \mathrm{msec}$. Slopes, in milliseconds/item, are given below this graph for searches for figure targets and for hole targets. On the right, mean RT is plotted against distance in depth of the target separately for the figure targets and the hole targets, to illustrate the target type $\times$ target distance interaction.

presence and set size was near significance $[F(1,8)=$ 4.66, $p=.063]$. Target distance was not significant, but there was a significant interaction between target distance and type $[F(1,8)=6.76, p=.032]$. As can be seen from Figure 6 , this interaction reflects a trend in favor of near targets for figures, but of far targets for holes. Importantly, there was no interaction between type and set size $[F(1,8)=0.26$, n.s. $]$.

Of the possible outcomes discussed in the introduction to Experiment 5, the second is the one that best fits the evidence. There was no evidence of an overall advantage for figures (as predicted by the first scenario) or holes (as predicted by the third scenario), and this was true whether one looked at main effects or slope differences. However, there was also no evidence that near targets were responded to more quickly in general. It is possible that this was due to the multiple depth planes involved for the set of items, since distractors could occur at the same plane as the target (see Figure 6).

As was mentioned in the introduction, an unavoidable consequence of using holes was that in all our experiments, the figure target and the hole target differed to- 
pologically. The importance of topology for perception is debated in the literature (e.g., Chen, 2001; Hecht \& Bader, 1998), and when a search asymmetry is found, we need to consider topology as a relevant dimension. Experiment 4 is particularly relevant for this hypothesis, because each item was either a small square figure with a circular hole or a small circle (without a hole). The fact that there was no interaction between type and set size fails to support the importance of topological differences in this type of visual search. More important for this article, this same result also fails to support an advantage for concavities in visual search.

\section{EXPERIMENT 6 Sparse Display and Target Distance}

Experiment 6 used the same design as that in Experiment 5, manipulating target distance, but with the sparse display introduced in Experiment 4. We expected to confirm the pattern of results found in Experiment 5 with the less regular display.

\section{Method}

The method and procedure were identical to those in Experiment 5. However, as in Experiment 4, items were presented within an invisible $4 \times 4$ matrix (see Figure 7). Twelve observers participated.

\section{Results and Discussion}

Mean RTs and slopes are shown in Figure 7. Overall error rate was $4.1 \%$. There were four within-subjects factors: target type (hole or figure), target presence (present or absent), the linear contrast of set size $(3,6$, or 9 items), and target distance (near, intermediate, or far). There was one between-subjects factor: order (search for a figure first or search for a hole first).

A mixed repeated measures ANOVA (type $\times$ presence $\times$ set size $X$ distance $X$ order) on RT yielded significant main effects of presence $[F(1,10)=39.68, p=.001]$ and of set size $[F(1,10)=112.19, p=.001]$. Responses were faster when the target was present, and RT increased linearly with increases in set size. Target distance was not significant, but there was a significant interaction between target distance and type $[F(1,10)=14.91, p=.003]$. There was also an interaction between set size and target presence $[F(1,10)=40.64, p=.001]$ but no interaction between type and set size $[F(1,10)=4.40$, n.s. $]$.

The results replicate those of Experiment 5. Once again, there was no evidence of an advantage for concavities (holes), and this was true whether one looked at main effects or slope differences. Also, near targets were not responded to more quickly in general, although this factor did interact with the type of the targets, as is shown in Figure 7.

\section{EXPERIMENT 7 Search Asymmetry}

This final experiment was fundamentally different from all the previous experiments. So far, we have compared figures and holes to see whether detection of concave contours is faster overall, and particularly for larger set sizes. We have failed to find support for either of these two predictions, which were based on the concavity hypothesis. Instead, our results support the part structure hypothesis that predicts no difference in the detection of concavities and convexities per se, providing that the stimuli do not differ in part structure.

However, a caveat to this conclusion is that our evidence in support of the part structure hypothesis is based on null effects - a lack of an overall advantage for detecting concave targets (holes) and a lack of an interaction between type and set size. It is, therefore, important to demonstrate that our experimental paradigm is sufficiently sensitive to detect a concavity advantage for stimuli similar to those presented in earlier visual search studies (e.g., Humphreys \& Müller, 2000). As was described in the introduction, both the concavity hypothesis and the part structure hypothesis predict a concavity advantage for these stimuli.

Experiment 7 comprised two conditions. In the first condition, the observers searched for a strictly convex target among distractors with both convexities and concavities, or vice versa. As in Humphreys and Müller (2000), the strictly convex shape looked like a barrel, and the shape with both convexities and concavities looked like an hourglass (see Figures 1 and 8). In the second condition, the observers searched for a strictly concave target (barrel-shaped holes) among distractors with both convexities and concavities (hourglass-shaped holes), or vice versa. This new condition was identical to the first condition, except that all of the shapes were now perceived as holes. Note that for both conditions, on a given trial, all the items were either figures or holes, and the target was defined by shape and not by disparity.

This experiment was conducted to try to reproduce a search asymmetry. All our experiments presented randomdot stereograms, rather than the high-contrast stimuli used by Hulleman et al. (2000) and Humphreys and Müller (2000), and it might be harder to detect a concavity advantage with such stimuli. We kept the parameters in Experiment 7 as similar as possible to those in Experiment 2. There was no need for a local background in Experiment 7 , because all the items shared the same location in depth, so the targets and the distractors differed only in shape. This is convenient, since it avoids the confounding effect of disparity that we had to deal with in the case of the search for a figure among holes and vice versa.

On the basis of Humphreys and Müller's (2000) results, we expected there to be an advantage for finding hourglass targets that increased with increasing set size. We did not expect any difference between the figures and the holes conditions, with respect to the interaction between shape and slope. With these stimuli, the extra complexity of the hourglass shape is present for both figures and holes (no three-way interaction). In terms of parts, for instance, the hourglass figure may be split into two convex subparts at its waist, whereas the hourglass hole has two convex parts pointing inward, and an observer may search for the presence of these elements. This means that our manipu- 

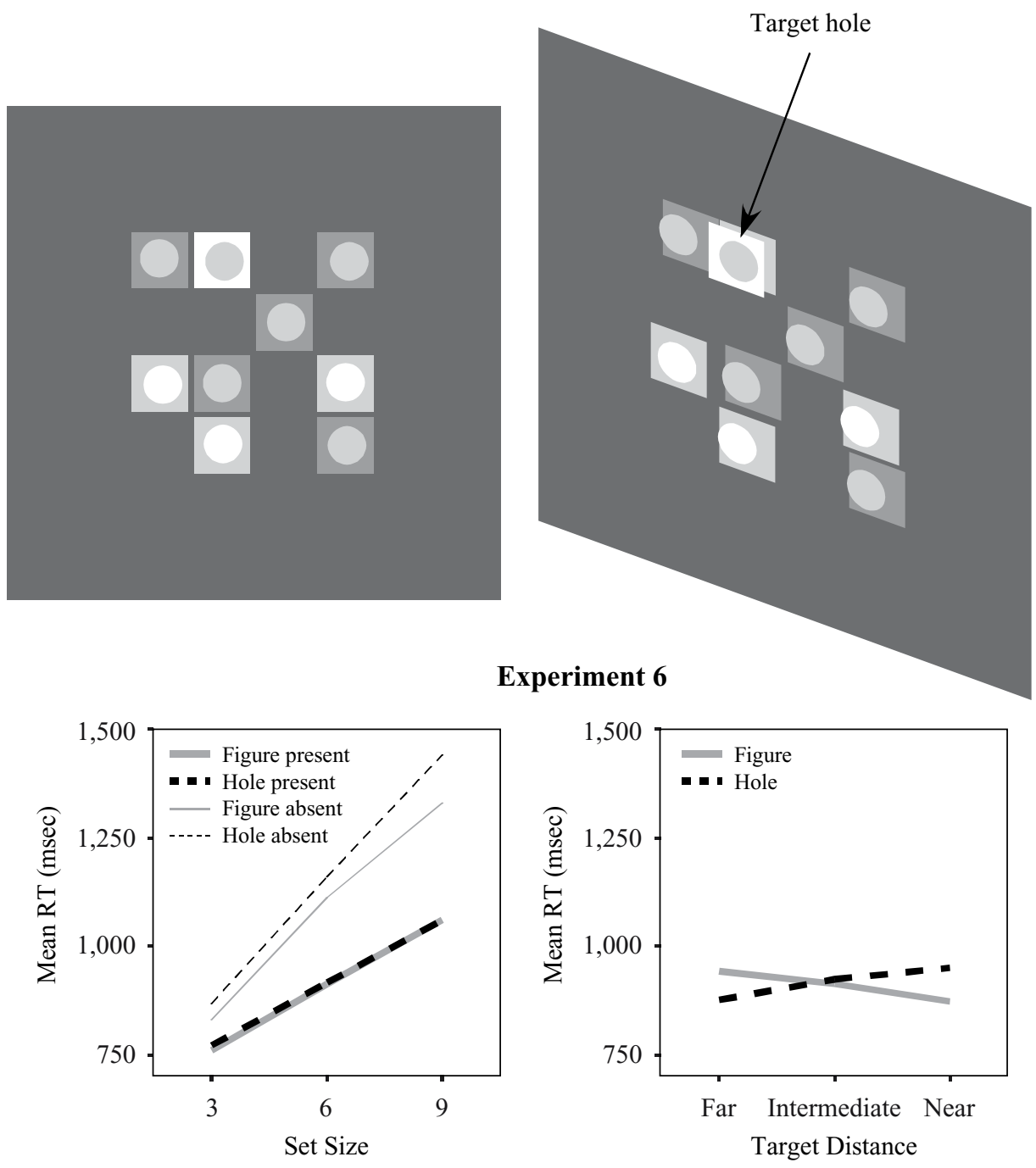

Figure slope: $49.81 \mathrm{msec} /$ item

Hole slope: $48.19 \mathrm{msec} /$ item

Figure 7. The stimuli (for a hole target) and results from Experiment 6. On the left, mean reaction time (RT) is plotted against set size for two types of targets (figure and hole) and for target-present and target-absent trials. The omnibus within-subjects standard error of the mean was $41.74 \mathrm{msec}$. Slopes, in milliseconds/item, are given below this graph for searches for figure targets and for hole targets. On the right, mean RT is plotted against distance in depth of the target separately for figure targets and hole targets, to illustrate the target distance $\times$ target type interaction.

lation of figure-ground (using holes) is fundamentally different from Humphreys and Müller's manipulation of figure-ground (creating two objects).

\section{Method}

The method and procedure were similar to those in Experiment 2, except that the shapes used were novel (see Figure 8). The design included the following factors: item type (holes or figures), target shape (barrel or hourglass), target presence (present or absent), and set size $(3,6$, or 9 items). There was one between-subjects factor: order (search for a barrel first or search for an hourglass first). Items were presented within an invisible $3 \times 3$ matrix. Fourteen observers participated.

\section{Results and Discussion}

Mean RTs and slopes are shown in Figure 8. Overall error rate was $7 \%$. There were four within-subjects factors: target type (holes or figures), target shape (barrel or hourglass), target presence, and the linear contrast of set size (3, 6, or 9 items). There was one between-subjects factor: order (search for a barrel first or search for an hourglass first).

We ran a mixed repeated measures ANOVA (type $x$ shape $X$ presence $\times$ set size $\times$ order) on RT. There were significant main effects of type $[F(1,12)=10.03$, $p=.008]$, presence $[F(1,12)=44.63, p=.001]$, shape 

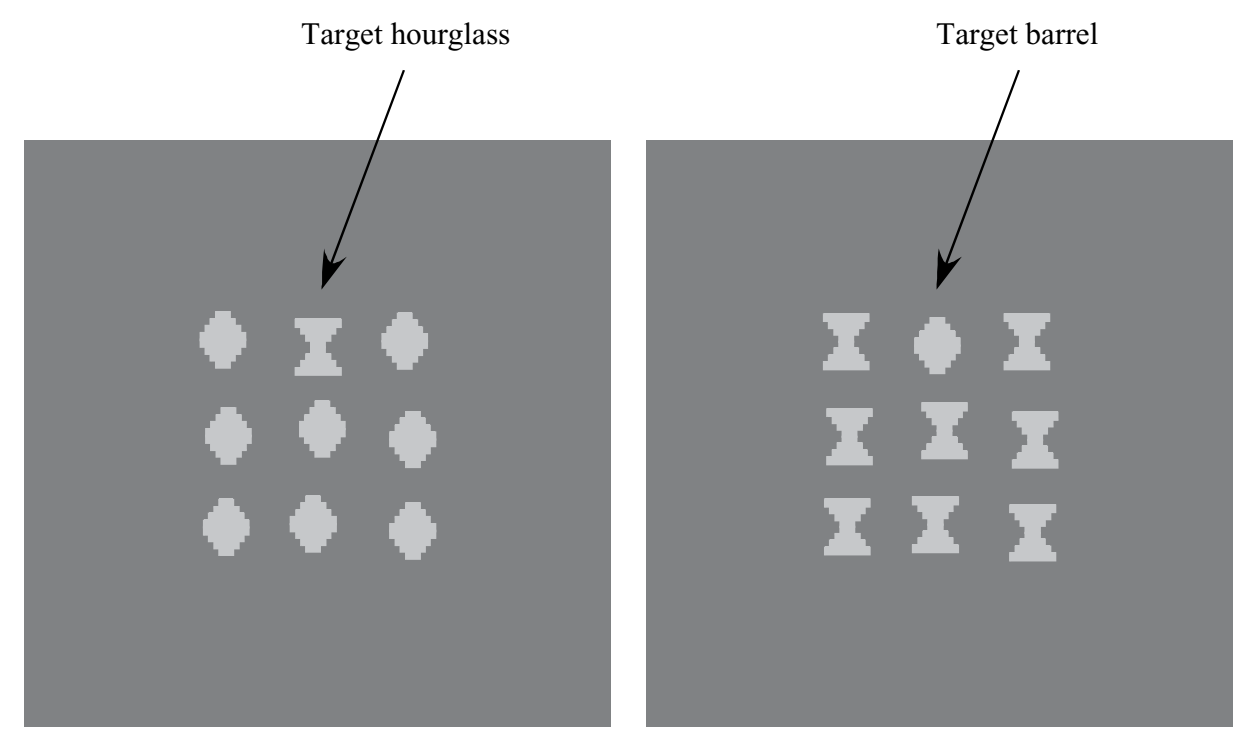

Experiment 7

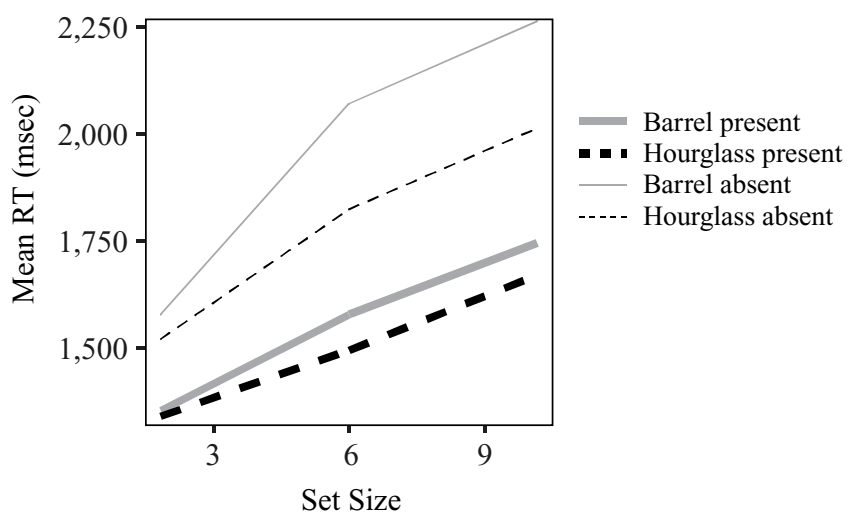

Barrel slope: $64.76 \mathrm{msec} /$ item

Hourglass slope: $54.99 \mathrm{msec} /$ item

Figure 8. The stimuli and results from Experiment 7. Mean reaction time (RT) is plotted against set size for two target shapes (barrel and hourglass) and for target-present and target-absent trials. The omnibus within-subjects standard error of the mean was 44.03 msec. Slopes, in milliseconds/ item, are given below this graph for searches for barrel targets and for hourglass targets.

$[F(1,12)=127.76, p=.001]$, and set size $[F(1,12)=$ $106.63, p=.001]$. Responses were faster when the items were figures, when the target was present, and when the target was the hourglass, and RT increased linearly with increases in set size. There were also significant interactions between set size and target presence $[F(1,12)=$ $18.51, p=.001]$, set size and shape $[F(1,12)=6.26, p=$ $.028]$, and set size and type $[F(1,12)=37.25, p=.001]$. These indicated that slopes were steeper when the target was absent, when the items were holes, and when the barrel was the target. Order was not significant, nor did it interact with any other factor.

The interaction between set size and shape was the most theoretically interesting, since it indicated a visual search asymmetry. Although displays with holes were harder in general, there was no significant higher order interaction involving set size and type. However, the criterion of less than $10 \mathrm{msec} /$ item for efficient search was not met by any of our conditions; slopes were over $50 \mathrm{msec} /$ item whether the target was an hourglass or a barrel. It is perhaps not surprising that search within a stereogram is harder than search within a high-contrast display such as that used in Experiment 1 in Humphreys and Müller (2000). Nevertheless, our results did replicate the concavity advantage, in the sense that there was a main effect of shape (faster responses to the hourglass target) and an interaction between shape and set size (shallower slopes for the hourglass target). Moreover, even the slope difference found 
by Humphreys and Müller was not large in absolute terms; for instance, in their Experiment 1, it was $3 \mathrm{msec} /$ item for the hourglass target and $8 \mathrm{msec} /$ item for the barrel target.

Displays with holes seem harder to process, which replicates Hulleman and Humphreys's (2005) findings, but the difference in slopes between shapes (barrel and hourglass) was similar for figures and holes. This might be seen as evidence of a similarity between the shapes of figures and holes. We prefer the interpretation that says that when convexities and concavities alternate along a contour, the change of sign causes a parsing into parts. The perceived parts would be different for an hourglass-shaped figure and an hourglass-shaped hole. However, what is critical is that these shapes would be more complex in terms of the perceived parts than either the barrel-shaped figure or the barrel-shaped hole. This experiment cannot separate these two possibilities (and was not designed to do so).

In summary, visual search may be relatively slow within a random-dot stereogram, but we found a concavity advantage for the hourglass target in Experiment 7, particularly for larger set sizes, consistent with previous findings. This study therefore confirms that our experimental paradigm was sufficiently sensitive to detect a concavity advantage when such an advantage was predicted by the part structure hypothesis.

\section{CONCLUSION}

In the visual search literature, it has been found that there is an advantage when searching for concave regions among convex shapes (i.e., search for concavities among convex shapes is efficient, whereas search for convex shapes among shapes with concavities is not). This has led some authors to hypothesize the concavity hypothesis: that the same edge is processed more efficiently when it is perceived as concave, perhaps because of specific detectors of concavities (Hulleman et al., 2000; Humphreys \& Müller, 2000). A similar suggestion about the salience of concavities was made by Barenholtz et al. (2003). However, these articles also acknowledged that concavities may be more salient, not in themselves, but because of their role in determining perceived part structure. It is generally agreed that when contour curvature changes sign, this information is used by the visual system to segment a shape. The part structure hypothesis suggests that it is the change from positive to negative contour polarity (i.e., from convexity to concavity), rather than the presence of negative curvature per se, that attracts attention. The available evidence from the previous visual search studies could not separate these two possibilities because strictly convex and strictly concave shapes were not compared directly. Instead, concavities were always presented as embedded within complex shapes (see Figure 1).

We started with the assumption that the part structure hypothesis was the better of the two accounts because this interpretation reconciles the visual search data with the data showing that when comparing vertex positions, performance is superior for convex contours (Bertamini \& Croucher, 2003; Bertamini \& Mosca, 2004; Gibson,
1994), and because no concavity advantage was found by Bertamini and Farrant (2005) using a change detection task.

The first six experiments tested performance when the target was strictly convex (a circular figure) or strictly concave (a circular hole). The results were complex with respect to the importance of some parameters, such as whether a preview of the background was shown and the relative distance in depth of the target and the distractors. However, with respect to the main goal of the article, we found no evidence of an advantage for responding to concave targets, as opposed to convex targets. This result is consistent with the predictions of the part structure hypothesis and provides evidence against the concavity hypothesis. Experiment 7 demonstrated that we could observe a concavity advantage using random-dot stereograms and shapes similar to those used in Humphreys and Müller (2000).

In conclusion, we have provided evidence against the hypothesis that concavities (unlike convexities) act as basic features and can be detected efficiently and preattentively because they are inherently more salient. Instead, our results support the hypothesis that concavities always play a central role in perception but that their importance is inextricably linked to their role in determining perceived part structure.

\section{REFERENCES}

Andersen, G. J. (1990). Focused attention in three-dimensional space. Perception \& Psychophysics, 47, 112-120.

Arnott, S. R., \& Shedden, J. M. (2000). Attention switching in depth using random-dot autostereograms: Attention gradient asymmetries. Perception \& Psychophysics, 62, 1459-1473.

Barenholtz, E., Cohen, E. H., Feldman, J., \& Singh, M. (2003). Detection of change in shape: An advantage for concavities. Cognition, 89, 1-9.

Bertamini, M. (2001). The importance of being convex: An advantage for convexity when judging position. Perception, 30, 1295-1310.

Bertamini, M., \& Croucher, C. J. (2003). The shape of holes. Cognition, 87, 33-54.

Bertamini, M., \& Farrant, T. (2005). Detection of change in shape and its relation to part structure. Acta Psychologica, 120, 35-54.

Bertamini, M., Friedenberg, J., \& Argyle, L. (2002). No within-object advantage for detection of rotation. Acta Psychologica, 111, 59-81.

Bertamini, M., \& LAwson, R. (2005). Testing inbuilt assumptions about convexity using a depth discrimination task. Manuscript submitted for publication.

Bertamini, M., \& Mosca, F. (2004). Early computation of contour curvature and part structure: Evidence from holes. Perception, 33, 35-48.

BIEDERMAN, I. (1987). Recognition-by-components: A theory of human image understanding. Psychological Review, 94, 115-147.

Chen, L. (2001). Perceptual organization: To reverse back the inverted (upside-down) question of feature binding. Visual Cognition, 8, 287-303.

Cohen, E. H., Barenholtz, E., Singh, M., \& Feldman, J. (2005).

What change detection tells us about the visual representation of shape. Journal of Vision, 5, 313-321.

Downing, C. J., \& Pinker, S. (1985). The spatial structure of visual attention. In M. I. Posner \& O. S. M. Marin (Eds.), Attention and performance XI (pp. 171-187). Hillsdale, NJ: Erlbaum.

Elder, J., \& Zucker, S. (1993). The effect of contour closure on the rapid discrimination of two-dimensional shapes. Vision Research, 33, 981-991.

ELDER, J., \& ZUCKER, S. (1998). Evidence for boundary-specific grouping. Vision Research, 38, 143-152. 
Fantoni, C., Bertamini, M., \& Gerbino, W. (2005). Contour curvature polarity and surface interpolation. Vision Research, 45, 1047-1062.

Feldman, J., \& Singh, M. (2005). Information along contours and object boundaries. Psychological Review, 112, 243-252.

Gibson, B. S. (1994). Visual attention and objects: One versus two or convex versus concave? Journal of Experimental Psychology: Human Perception \& Performance, 20, 203-207.

He, Z. J., \& NAKaYAma, K. (1992). Surfaces versus features in visual search. Nature, 359, 231-233.

Hecht, H., \& BADER, H. (1998). Perceiving topological structure of 2-D patterns. Acta Psychologica, 99, 255-292.

HoffMan, D. D., \& Richards, W. (1984). Parts of recognition. Cognition, 18, 65-96.

Hulleman, J., \& Humphreys, G. W. (2005). Differences between searching among objects and searching among holes. Perception \& Psychophysics, 67, 469-482.

Hulleman, J., Te Winkel, W., \& Boselie, F. (2000). Concavities as basic features in visual search: Evidence from search asymmetries. Perception \& Psychophysics, 62, 162-174.

Humphreys, G. W., \& Müller, H. (2000). A search asymmetry reversed by figure-ground assignment. Psychological Science, 11, 196-201.

Kanizsa, G. (1979). Organization in vision. New York: Praeger.

Kanizsa, G., \& Gerbino, W. (1976). Convexity and symmetry in figure-ground organization. In M. Henle (Ed.), Vision and artifact (pp. 25-32). New York: Springer.

KaWAbe, T., \& MiUra, K. (2004). Perceptual grouping in shape from shading. Perception, 33, 601-614.

KoENDERINK, J. J. (1984). What does the occluding contour tell us about solid shape? Perception, 13, 321-330.

Marr, D., \& Nishihara, H. K. (1978). Representation and recognition of the spatial organization of three-dimensional shapes. Proceedings of the Royal Society of London, 200, 269-294.

Nakayama, K., \& Shimojo, S. (1992). Experiencing and perceiving visual surfaces. Science, 257, 1357-1363.

Nelson, R., \& Palmer, S. E. (2001). Of holes and wholes: The perception of surrounded regions. Perception, 30, 1213-1226.

O'Toole, A. J., \& WalKer, C. L. (1997). On the preattentive accessibility of stereoscopic disparity: Evidence from visual search. Perception \& Psychophysics, 59, 202-218.

Palmer, S. E. (1999). Vision science: Photons to phenomenology. Cambridge, MA: MIT Press.

PelLi, D. G. (1997). The Video Toolbox software for visual psychophysics: Transforming numbers into movies. Spatial Vision, 10, 437-442.

RichaRds, W., KoENDERINK, J. J., \& HoFFMAN, D. D. (1985). Inferring $3 D$ shapes from $2 D$ codons (AI Memo No. 840 ). Cambridge, MA: MIT, Computer Science \& Artificial Intelligence Laboratory.

Rock, I. (1983). The logic of perception. Cambridge, MA: MIT Press

Rosenholtz, R. (2001). Search asymmetries? What search asymmetries? Perception \& Psychophysics, 63, 476-489.
RuBIN, E. (1921). Visuell wahrgenommene Figuren. Copenhagen: Gyldendalske Boghandel.

Singh, M., \& Hoffman, D. D. (2001). Part-based representations of visual shape and implications for visual cognition. In T. F. Shipley \& P. J. Kellman (Eds.), From fragments to objects: Grouping and segmentation in vision (Advances in Psychology, Vol. 130, pp. 401-459). New York: Elsevier.

Treisman, A., \& Souther, J. (1985). Search asymmetry: A diagnostic for preattentive processing of separable features. Journal of Experimental Psychology: General, 114, 285-310.

\section{NOTES}

1. Elder and Zucker $(1993,1998)$ found that only when contours formed a closed region did the difference between concave and convex stimuli lead to efficient visual search. This evidence indirectly supports the importance of convexities and concavities, as well as closure, because the sign of contour curvature is undefined without closure.

2. Note that Cohen, Barenholtz, Singh, and Feldman (2005) found a concavity advantage, using the same task as that in Bertamini and Farrant (2005) but different stimuli. This advantage may be due to the location of the convexities in their stimuli: farther away from the inside of the object with respect to the concavities. The discrepancy between the two sets of results needs to be resolved by further empirical research.

3. Convexity influences figure-ground organization (Rubin, 1921). Empirical support for this was found by Kanizsa and Gerbino (1976). Bertamini and Lawson (2005) recently confirmed that responses are faster to locate a convex figural region than a concave figural region. This evidence is also problematic for the concavity hypothesis because it suggests that concave regions are not more salient per se.

4. We also believe that the part structure hypothesis is consistent with the role of surfaces in visual search, as demonstrated by $\mathrm{He}$ and $\mathrm{Na}$ kayama (1992; Nakayama \& Shimojo, 1992). A change of contour polarity is informative about solid shape (Koenderink, 1984, Richards et al., 1985), and this may be why it is easier to find such occurrences.

5. We also conducted an experiment with only one local background (not included here for reasons of space) with either a hole through it or a figure over it. So that disparity would not increase with distance, the hole could appear only when the local background was close to the overall background. This experiment confirmed that there was no hole advantage, and indeed, there was a small figure advantage. However, the figure advantage could have originated from an advantage for the nearer target or even to the extra surface present in the case of the figure (but not the hole).

(Manuscript received September 24, 2004; revision accepted for publication August 11, 2005.) 